\title{
Polymer Optical Fiber Intensity-Based Sensor for Liquid-Level Measurements in Volumetric Flasks for Industrial Application
}

\author{
D. S. Montero and C. Vázquez \\ Electronics Technology Department, Carlos III University of Madrid, Avenida de la Universidad 30, Leganés, 28911 Madrid, Spain \\ Correspondence should be addressed to D. S. Montero, dsmontero@ing.uc3m.es
}

Received 22 March 2012; Accepted 8 May 2012

Academic Editors: T.-S. Chen, J.-F. Myoupo, L. Reggiani, and Y.-C. Wang

Copyright () 2012 D. S. Montero and C. Vázquez. This is an open access article distributed under the Creative Commons Attribution License, which permits unrestricted use, distribution, and reproduction in any medium, provided the original work is properly cited.

\begin{abstract}
A low-cost intensity-based polymer optical fiber (POF) sensor for liquid detection applied to volumetric flasks is presented. Experimental results demonstrate the viability of the POF-based sensor system in a high-accuracy liquid level measurement scenario. Moreover, a wireless mesh sensor network based on ZigBee specification protocol to address multiplexed POF-based sensor is also developed. Experimental results demonstrate the feasibility to address a high number of optical sensors in an industrial process framework by means of this low-cost wireless solution.
\end{abstract}

\section{Introduction}

Fiber-optic sensors exhibit a set of very attractive characteristics, including immunity to electromagnetic interference, small-sized capability, resistance to hostile environments that may comprise hazardous chemicals or of any other kind, geometric versatility, ruggedness, sensor multiplexing, and distributed sensing over a single fiber. There are numerous realizations of fiber-optic sensors but one extensively investigated transducing mechanism in optical sensing applications is the intensity modulation of the propagating light. Approaching simple configurations, intensity sensors modulate the optical power loss as the physical magnitude changes, thus providing the measurement as an optical intensity modulation signal. Intensity-based fiber-optic sensors have been demonstrated in literature to be very reliable, simple in concept, easily made selective to specific measurand, easily integrated in optical networks by means of different multiplexing techniques, and cost-effective sensing approach for a wide range of applications. In particular, if a flammable environment is a critical concern for industrial sensor applications, an intensity-based fiber-optic sensor is one of the best candidates. Moreover, these fiber-optic sensors have attractive properties for liquid-level measurements in practice. By using reflective intensity modulation to represent a change in the surrounding material, submersion or flooding can be monitored. These applications can be carried out in oil tanks, flood areas, and underground $[1,2]$.

It should be outlined that in the optical sensing field, fiber-optic sensors can be constructed using polymer optical fibers (POFs) rather than silica-based versions, both singlemode (SMF) and multimode (MMF), because POFs have large numerical apertures, simple alignment to optical devices, high coupling efficiency, more flexibility, and lower cost. These are some reasons why new POF-based sensors have appeared and are still appearing, most of them based on optical power intensity detection, for monitoring liquid level $[3,4]$.

Optical fiber sensors for liquid level measurement have been extensively studied. Most liquid-level optical sensors are discrete or point-level sensors [4], while others allow continuous measurements [5]. In some cases, the sensor is a sensitive element by detecting radiation losses in bends [6] and reflective intensity variations [1] because of surrounding material changes. In these cases the fiber is submerged slightly in the liquids to indicate its presence thus performing intrusive sensors. Moreover, to increase the sensor sensitivity, configurations with bends plus cladding removal and partially polished fiber core have been studied, such as the work reported in [3]. A nonfiber specific attached head such as 
a prism [7] has also been analyzed but based on total internal reflection (TIR). Intrusive schemes based on Fiber Bragg Grating (FBG) and Long Period Fiber Grating (LPG) sensors have also been studied [8]. In other systems, the measurement is nonintrusive taken by the light reflection on the surface of the liquid [9].

Level detection plays an important role in commercial and technological applications. Different methods such as mechanical, capacitive, inductive, ultrasonic [10], acoustic [11], or optical can be implemented. Typically, mechanical and ultrasonic methods are used to detect the level of solid materials that are in the form of dust whereas capacitive and optical methods give better results in detecting the level of fluids. On the other hand and focusing on industrial processes, volumetric flasks are used in analytical chemistry for making up solutions or liquids, normally distilled water, to a known volume. They are made of glass or plastic and consist of a flat bottomed bulb with a long neck, usually fitted with a stopper. The glass or plastic is generally colorless but may be amber colored for handling light-sensitive compounds such as silver nitrate or vitamin $\mathrm{A}$, for example. In addition to this, the calibration mark consists of a single ring graduation mark drawn over the long neck of the flask, and the measurements are taken considering the liquid meniscus, defined as the curvature of the liquid in a narrow container. The level of concave (downward curving) liquid surfaces is read at the bottom of the meniscus. However, the procedures to determine the liquid (or solution) level are often based on visual inspections. Typically the meniscus can be made clearer by holding a buret card or a white card behind the neck just below the line of sight to contrast the line between the glassware markings and the liquid level. It is not easy to automate this procedure of measurement.

Additionally, the possibility of multiplexing these POF intensity-based sensors is presented. The sensor network topology performs a wireless mesh network in which the radio nodes (or access points (Aps)) are organized in a mesh topology offering reliability and redundancy. So if a node can no longer operate, the rest of the nodes can still communicate with each other, directly or through one or more intermediate nodes. This wireless mesh network can be implemented in different wireless technologies, and ZigBee specification protocol (IEEE 802.15.4-2003 standard) can provide the main building block of ubiquitous networks for low-rate Wireless Personal Area Networks (WPANs), as sensing applications usually require [12]. Nevertheless, the whole system and how the different sensors are interconnected can be seen as a proof-of-concept for the measurement of any physical magnitude (mechanical, thermal, flow, chemical, radiation, etc.) with regard to other intensity-based POF sensors.

In this work a nonintrusive POF intensity-based fiberoptic sensor for high-accuracy liquid level measurement in industrial processes is presented with applications in volumetric flasks which are used in analytical chemistry for making up solutions or liquids to a known volume. A proofof-concept of a low-cost wireless network based on ZigBee protocol for addressing a determined number of optical sensors is also demonstrated. This wireless mesh sensor network offers a relatively inexpensive, scalable, robust, and a low-power consumption solution. In addition, a graphical interface for the management of the sensor system is presented.

\section{Sensor Description and Measurements}

The POF-based fiber-optic sensor developed performs an intrinsic and a nonintrusive measuring device. Light is launched into an emitter fiber thus illuminating the volumetric flask in which the liquid is immersed and received into a reciprocally placed (same longitudinal axes) optical fiber. The operation consists of a classical optoelectronic level detection method in which the light, between the emitter and receiver fiber, is cut off. Both POFs are located at a certain height of the flask depending on the nominal liquid volume to measure considering also the liquid meniscus, by means of a simple relation between volume and height, and taking into account the flask geometry. The optical measuring principle of the level detection system is shown in Figure 1(a). A photograph of a sensing head based on this principle is depicted in Figure 1(b). Sensing head was manufactured with lowcost commercial $980 \mu \mathrm{m}$ core diameter step-index PMMA (polymethylmethacrylate) POFs. These fibers have core and cladding refractive indices of $n_{\mathrm{co}}=1.492$ and $n_{\mathrm{cl}}=1.417$, respectively, and numerical aperture of 0.47 .

Let us explain the detection principle. If there is no liquid between the emitter fiber and the receiver fiber, light that is being emitted can fall on the receiver fiber. As liquid level rises, it begins to block the detection area of the receiver fiber, depending on its numerical aperture (NA), and some percent of the light reflects from the liquid surface. When the liquid level reaches the height of interest, given by the liquid meniscus and where both fibers are placed, the detection area of the receiver fiber is completely blocked and the emitted light is scattered from the liquid surface. Accordingly, no optical power is received at the detection stage. When the liquid level rises to a higher extent, the receiver optical power rises as emitted light falls on the detection area by passing through the liquid. Consequently, in every case, the amount of light that is transmitted to the receiver fiber depends, basically, on the output power of the emitter one, the light permeability coefficient of the medium (liquid, air, etc.), and the distance between the emitter and the receiver fibers, but only producing changes in the detected optical power, the second aforementioned condition.

The set-up block diagram of the level detection sensor is illustrated in Figure 2(a). At the reception stage the signal conditioning is implemented using low-cost commercial capacitors, resistors, and amplifiers before the microprocessor stage. As the meniscus condition can be easily reached from one drop of liquid to the following added at the volumetric flask. Alternatively, it is possible to locate another sensing head at a lower height of the flask performing two filling modes, continuous (or fast) filling, and drop filling (i.e., drop by drop). The switch between these two modes can be easily implemented by means of a microprocessor by connecting the sensing heads to the master electronic board. 


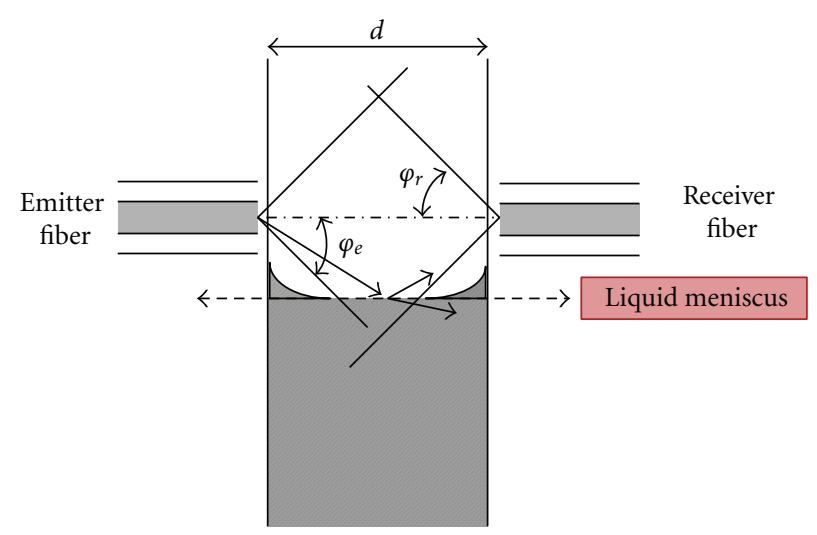

(a)

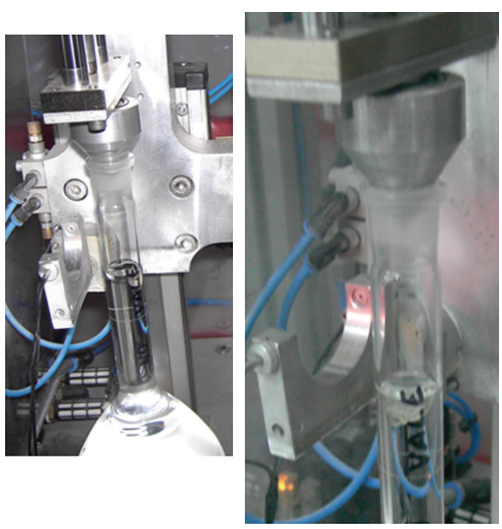

(b)

FIgure 1: (a) Optical diagram of the level detection method applied to volumetric flasks. (b) Photograph of the sensing head.

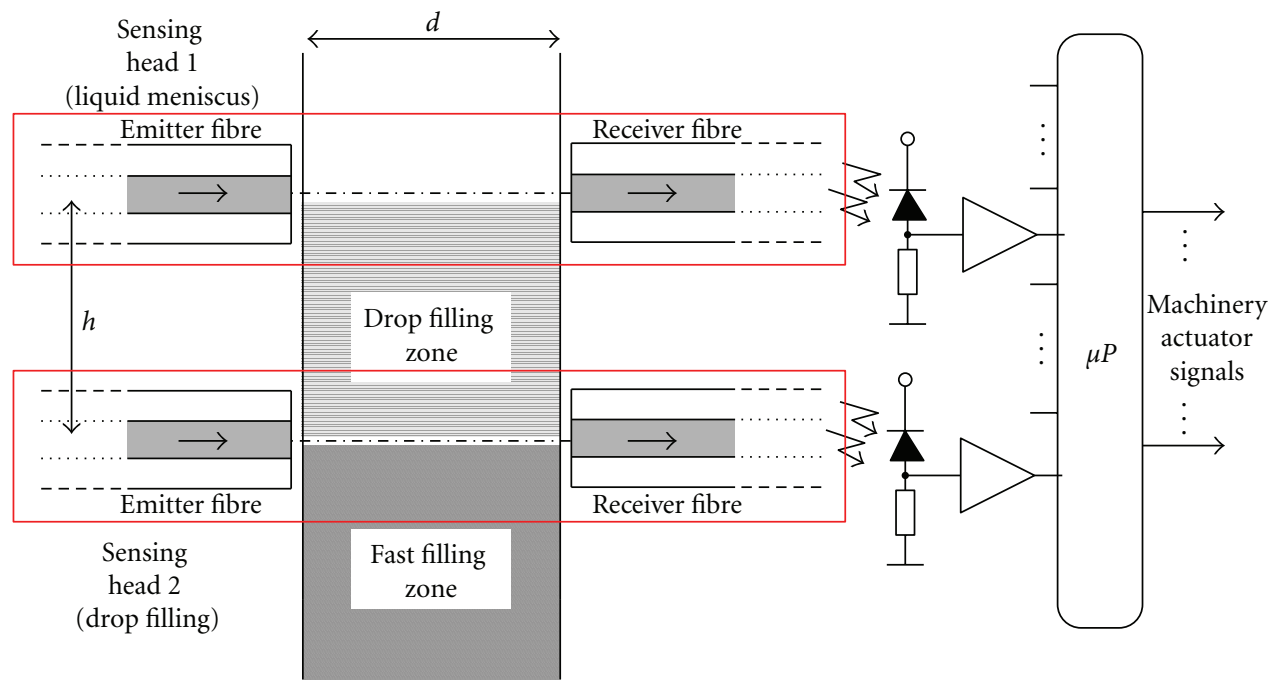

(a)

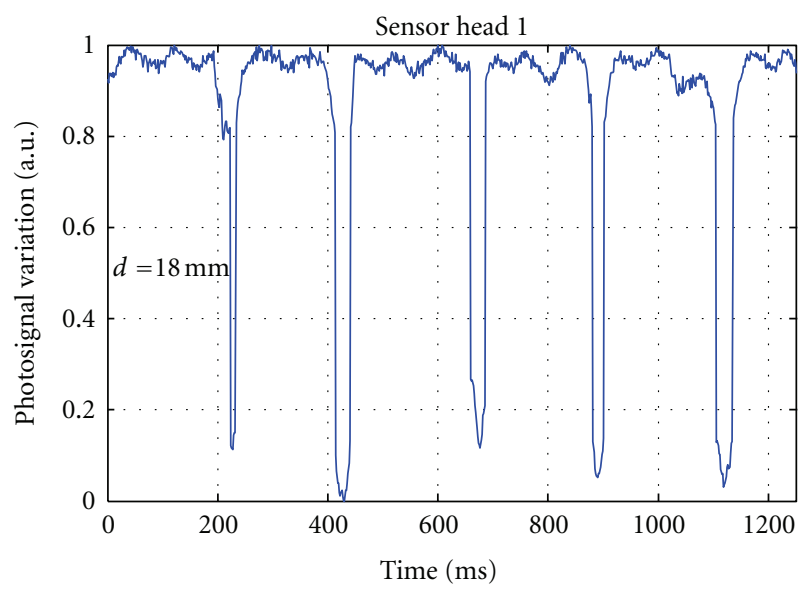

(b)

Figure 2: (a) Set-up diagram of the level detection sensor applied to volumetric flasks. (b) Normalized photosignal variation versus time in drop filling regime for distilled water. 


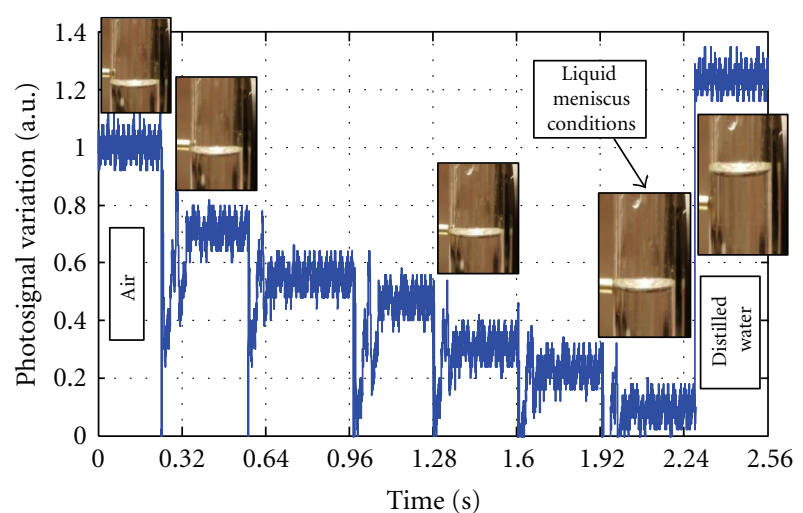

FIGURE 3: Normalized photosignal response of the sensor system versus time in drop filling regime, for distilled water. Inset: photos of liquid level for different scenarios.

Some other circuits can be connected to the output of the microprocessor according to system requirements. The parameter $h$, see Figure 2(a), which defines the distance between the two sensing heads, can be optimized to perform a fast automated volumetric flask filling. In the set-up implemented, this parameter takes the value of $1.5 \mathrm{~cm}$. Finally, operating in the drop-filling zone, the volumetric flask filling is made drop by drop to obtain an accurate measurement of the total volume contained by the flask. The measured normalized photosignal variation for the sensing head 1 is shown in Figure 2(b). The response for 5 drops is shown, in drop filling regime for distilled water. When liquid level reaches the desired height (in which the sensor head is located), a mean variation of $-9.6 \mathrm{~dB}$ in the optical power impinging the receiver fiber is detected, and liquid meniscus condition is fulfilled. It should be noted that the aforementioned sensitivity is performed just by adding a single liquid drop from a previous scenario of liquid level.

Figure 3 deploys the temporal response of the POF sensor system (sensor head 1) within the drop filling zone, six drops before reaching the desired volume of distilled water. Each normalized decreasing photosignal detected level corresponds to a new drop added inside the volumetric flask. Finally, it can also be seen that the received optical power is higher when distilled water is present at the sensor height compared to the case when the medium is air. By applying a liquid with a different refractive index than that of the air $\left(n_{\text {air }}=1\right)$, Fresnel losses change due to the different amount of refracted light within the flask. And this fact will contribute to a higher light coupling into the receiver fiber and so the optical power impinging the photodetector at reception.

Figure 4 shows the optical power at reception for different measurement scenarios. Conditions for each scenario are given in the enclosed table. Scenario SC0 involves the case when no volumetric flask is placed between the emitter and receiver fiber. All light is air-propagated, and a detected optical power at the end of the receiver of $-19.3 \mathrm{dBm}$ is measured. Sensor head insertion losses are found to be $\sim 4.5 \mathrm{~dB}$. Once the volumetric flask is placed between both fibers, a decrease of $0.6 \mathrm{~dB}$ in optical power is measured. This fact

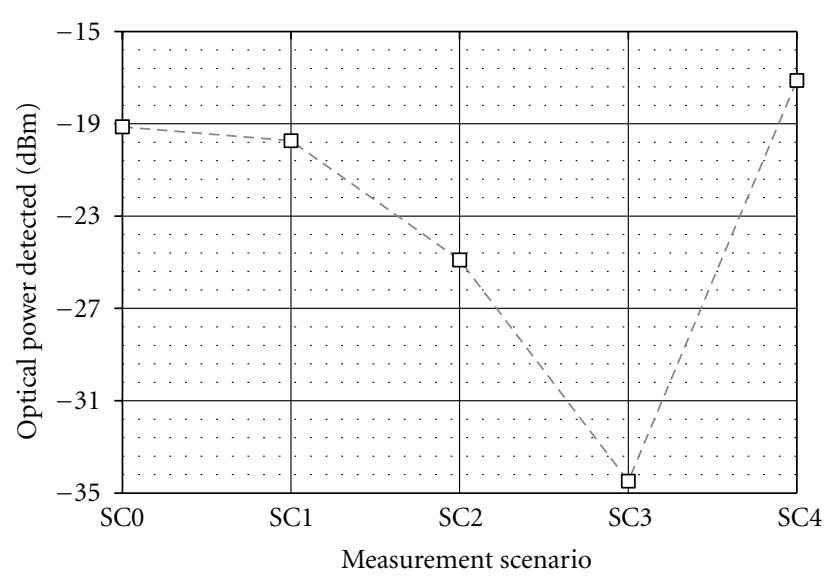

FIGURE 4: Optical power detected at the end of the receiver fiber for the different measurement scenarios, in case of distilled water. SC0: air gap between emitter and receiver fiber. SC1: volumetric flask is placed between both fibers of the sensing head. SC2: liquid level close to where the emitter fiber is placed. SC3: liquid meniscus condition. SC4: liquid interface between emitter and receiver fiber.

is illustrated in Figure 4 scenario 2 (SC1). As liquid level rises and gets closer to the emitter fiber, $\mathrm{SC} 2$, it begins to block the detection surface of the receiver fiber, and some percent of the light reflects from the liquid surface. Consequently, less power is detected at reception although air interface between both fibers still stands. A nominal value of $-24.9 \mathrm{dBm}$ was measured for this scenario. It should be mentioned that measurement scenario SC2 corresponds to the starting time represented in Figure 3. When liquid meniscus condition at the sensing head is fulfilled (SC3), most of the acceptance area of the receiver fiber is completely blocked and light reflects from the liquid surface. A mean variation of $-9.6 \mathrm{~dB}$ in the optical power impinging the receiver fiber is detected with regard to previous scenario SC2. 5 measurements were computed for this scenario (our case of interest) with standard deviation of $<7 \%$ from the nominal value. Although this value seems to be high it does not represent a shortcoming for opening up the election of decision thresholds of the received photosignal in the microprocessor at the optoelectronics stage. Finally, a distilled water interface between both fibers emerges, light passes through the liquid, and a higher power is detected at reception; see SC4.

\section{Wireless Mesh Sensor Network}

A wireless mesh network is a type of mobile wireless network that is decentralized, relatively inexpensive, and very reliable and resilient. It is built of peer radio devices that do not have to be cabled to a wired port like traditional Wireless Local Area Networks (WLANs) access points (APs) do. The most important feature that distinguishes wireless mesh networks from other wireless topologies is robustness. This means that 


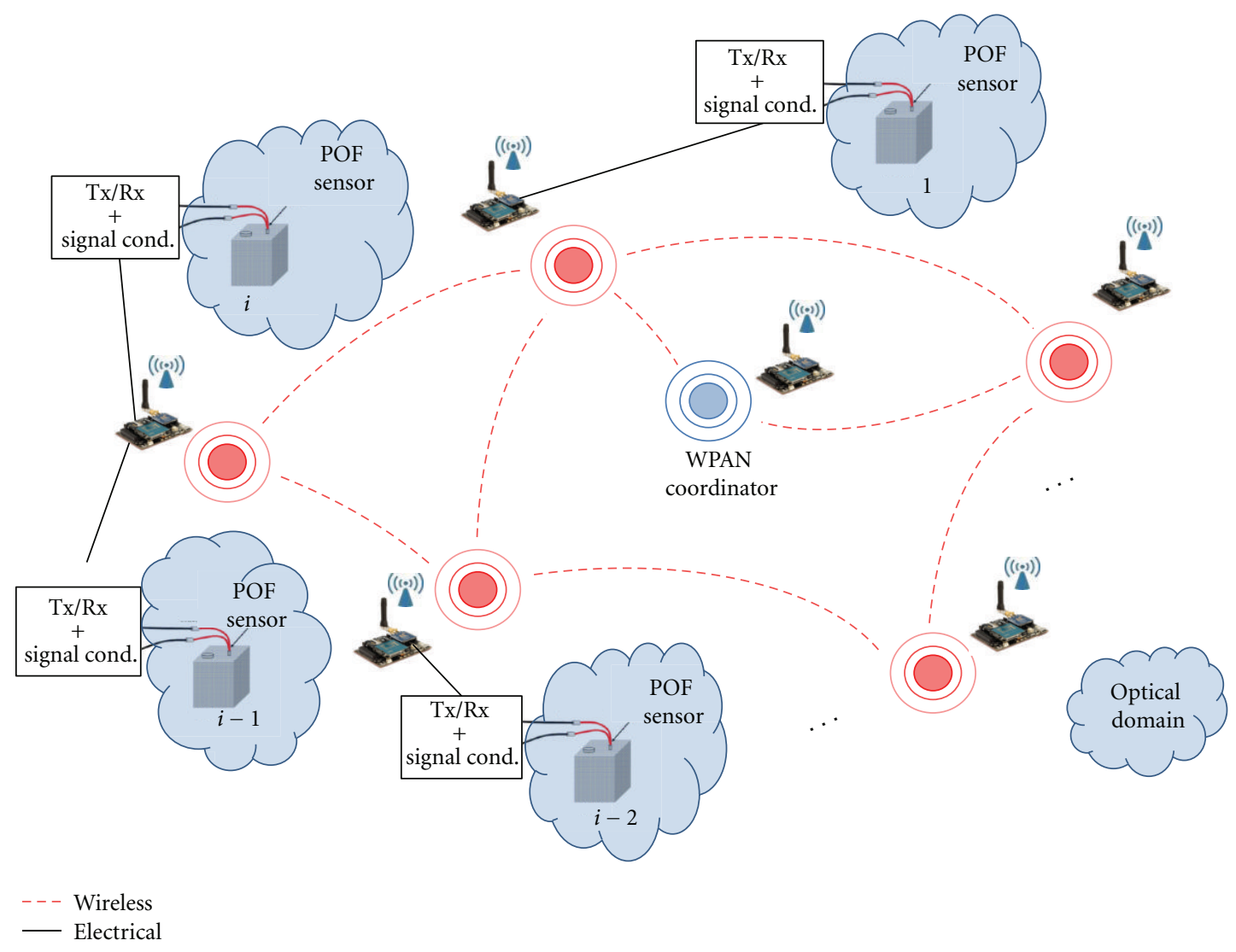

FIgURE 5: Diagram of a generic wireless mesh sensor network.

if one node drops out of the network, due to hardware failure or any other reason, its neighbors find another route. Nodes act as routers to transmit data from nearby nodes to peers that are too far away to reach in a single hop, resulting in a network that can span large distances (typically $100 \mathrm{~m}$ outdoor and $30 \mathrm{~m}$ indoor). Wireless mesh sensor networks merge advantages of wireless mesh networks and wireless sensor networks, especially on scalability, robustness, and balanced power consumption. Accordingly, these latter are attracting more and more attentions from industry and academic communities as a possible way to improve the reliability and throughput of sensor networks and support the node mobility [13]. Figure 5 depicts a diagram of a generic wireless mesh sensor network.

On the other hand, ZigBee protocols are intended for use in applications requiring low data rates and low power consumption (individual devices must have a battery life of at least two years to pass ZigBee certification). Some examples are wireless light switches with lamps, in-home displays, consumer electronics, or in-home sensing systems via shortrange radio needing low rates of data transfer. The technology defined by the ZigBee specification is intended to be simpler and less expensive than other WPANs, such as Bluetooth or Wi-Fi. From the analysis between different wireless standards shown in Table 1, ZigBee is targeted at radiofrequency applications that require low data rate, long battery life, and secure networking.
3.1. Experimental Results and Network Performance. The proposed wireless mesh POF-based sensor network is evaluated in this section. A 4-node network has been implemented, in which 3 Xbee modules (these modules allow to communicate wirelessly using ZigBee protocol operating at a radiofrequency of $2.4 \mathrm{GHz}$ for worldwide deployment) have been used as ZigBee End Devices (ZED) and 1 Xbee module has been used as ZigBee Coordinator (ZC). Each module has been placed in an Arduino Diecimila board containing a microcontroller board based on the ATmega 168 microprocessor and also performing 6 analog inputs (i.e., 6 sensors could be connected to each ZED) with a 10-bit ADC converter each. These ZigBee protocol modules support a full-duplex non-beacon-enabled network in which an unslotted CSMA/CA (Carrier Sense Multiple Access with Collision Avoidance) channel access mechanism is used. This allows for heterogeneous networks in which some devices receive continuously, while others only transmit when an external stimulus is detected. It should be mentioned that it is estimated an average response timing of less than $10 \mathrm{~ms}$ per sensor per module from the sensor data acquisition to the data updating at the user interface. Attending time span from Figure 3, this latter fact can be seen as a real-time fluidlevel monitoring.

As a proof-of-concept of the sensor network proposed in this paper, a laboratory testbed including six POF intensitybased optical sensors has been implemented constituting 
TABLE 1: Comparison between different wireless standards.

\begin{tabular}{llll}
\hline & \multicolumn{2}{l}{ Wireless communication standard specifications } & Wi-Fi \\
& ZigBee & Bluetooth & 802.11 \\
Standard & 802.15 .4 & 802.15 .1 & Up to $54 \mathrm{Mbps}$ \\
Data rate & $250 \mathrm{kbps}$ & $1 \mathrm{Mbps}$ & $>400 \mathrm{~mA}$ \\
Power consumption (Tx mode) & $35 \mathrm{~mA}$ & $40 \mathrm{~mA}$ & $20 \mathrm{~mA}$ \\
Current consumption (sleep mode) & $3 \mu \mathrm{A}$ & $200 \mu \mathrm{A}$ & $\sim 20 \mathrm{~m}$ (indoor) \\
& $\sim 30 \mathrm{~m}$ (indoor) & $\sim 30 \mathrm{~m}$ (indoor) & \\
Range & $\sim 100 \mathrm{~m}$ (outdoor) & $\sim 100 \mathrm{~m}$ (outdoor) & Point-to-multipoint \\
& Peer-to-peer & & Point-to-multipoint \\
Topology & Point-to-point & & \\
& Point-to-multipoint & & Data transfer \\
Applications & Device interconnection & Device interconnection & Data transfer \\
& Illumination & Audio & \\
& Sensing & Control \& Automation &
\end{tabular}

TABLE 2: Performance of the different liquid level detection POF intensity-based optical sensors.

\begin{tabular}{|c|c|c|c|c|c|c|}
\hline & \multicolumn{2}{|r|}{ ZED 1} & \multicolumn{2}{|r|}{ ZED 2} & \multicolumn{2}{|l|}{ ZED 3} \\
\hline & Sensor $1^{\mathrm{a}}$ & Sensor $2^{\mathrm{b}}$ & Sensor $1^{\mathrm{c}}$ & Sensor $2^{\mathrm{d}}$ & Sensor $1^{\mathrm{e}}$ & Sensor $2^{f}$ \\
\hline Linearity & - & 0.9877 & - & 0.9777 & 0.9649 & - \\
\hline Sensitivity & $5.3 \%$ F.S. & $1.3 \mathrm{~dB} /$ discrete level & $12 \%$ F.S. & $0.35 \mathrm{~dB} /$ discrete level & $0.85 \mathrm{~dB} /$ discrete level & $1.7 \%$ F.S. \\
\hline Measurements standard deviation & $<9 \%$ & $<8 \%$ & $<5 \%$ & $<5 \%$ & $<12 \%$ & $<2 \%$ \\
\hline
\end{tabular}

Linearity is given in terms of the linear regression coefficient; F.S.: full-scale.

${ }^{a}$ Air/water/oil detection, $R=7 \mathrm{~mm}, \varepsilon=0.23 \mathrm{~mm}$, self-referencing sensor; ${ }^{\mathrm{b}}$ discrete multipoint fuel level sensor, stripped fibre, gauge-shape bend.

${ }^{\mathrm{c}}$ Air/water/oil detection, $R=25 \mathrm{~mm}, \varepsilon=0.23 \mathrm{~mm}$, self-referencing sensor; ${ }^{\mathrm{d}}$ discrete multipoint fuel level sensor, polished fibre, no bend.

${ }^{\mathrm{e}}$ Discrete multipoint fuel level sensor, stripped fibre, helicoidal bend; fair/water/oil detection, $R=60 \mathrm{~mm}, \varepsilon=0.21 \mathrm{~mm}$, self-referencing sensor.

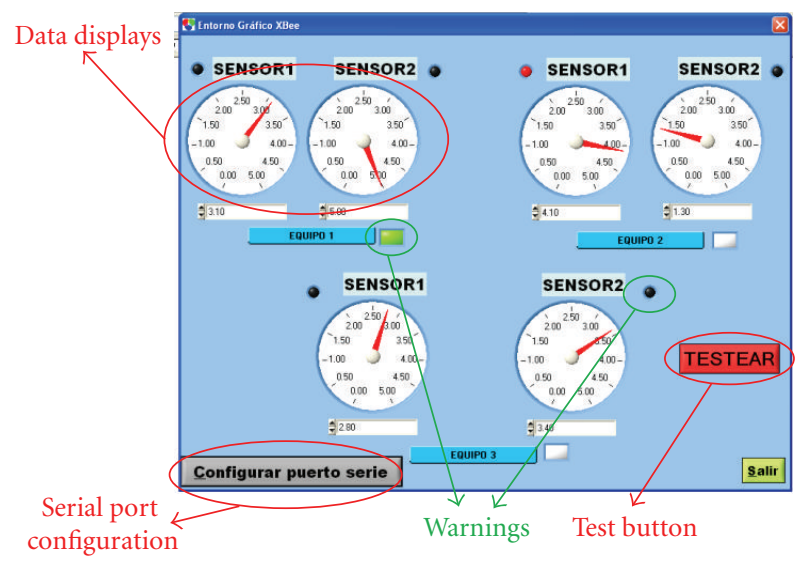

Figure 6: Users management main console.

a wireless mesh 6-sensor network, in which each ZED Xbee module performs a 2-POF sensor node [14]. Nevertheless, for this proposed 3-node (i.e., 3 ZEDs) wireless sensor network a maximum of 18 optical sensors could remotely be addressed. Table 2 shows the performance of the complete set of POF-based optical sensors. The latter have been developed and manufactured for liquid-level detection measurement following the principle of operation of radiation losses in bends in optical fibers $[3,6]$. It should be noted that sensor sensitivities are given in both optical power variation per discrete level in the case of discrete multipoint sensors or in terms of the full-scale sensitivity for the case of intrinsically self-referencing sensors.

In addition, a friendly graphical interface in LabWindows platform for the management of the complete system has also been developed. The optical sensor outputs can be continuously monitored and displayed. Different user functionalities have been performed such as the possibility of a serial port configuration, a test button to enable data acquisition from all POF sensors with only one click, or different system warnings, which give an idea to the user of how the whole system is performing. Figure 6 shows a snapshot from the main console for user management.

\section{Conclusions}

A low-cost intensity-based polymer optical fiber sensor for liquid level detection applied to volumetric flasks is 
presented. The operation principle consists of a classical optoelectronic level detection method in which the light, between the emitter and receiver fibre, is cut off. The received optical power dramatically decreases when liquid meniscus condition is fulfilled allowing to exactly distinguish the volume of liquid contained. The advantage of this method is that optical properties of the liquid do not have significant effects on level detection phenomenon. Furthermore it can be exten-ded in detecting levels of water and inflammable fluids (gasoline, fuel oil, alcohol, etc.). Furthermore, the associated optoelectronics can make this process to be fully automated.

Experiments have been carried out with distilled water as fluid. Experimental results demonstrate the viability of the POF-based sensor system in a high-accuracy liquid level measurement scenario. Near liquid meniscus condition (i.e., six drops of fluid before) the system performs different sensitivities as light becomes more reflected from fluid's surface, and standard deviations below 7\% from the nominal values are obtained. Consequently, decision thresholds of the received photosignal in the microprocessor at the optoelectronics stage can be easily implemented to perform the system automation.

Taking advantage of the ease of integration in optical networks by intensity-based fiber-optic sensors, a proof-ofconcept of a 4-node wireless mesh sensor network consisting of six polymer optical fibre (POF) intensity-based sensors is demonstrated. As sensing applications usually require low data rates and low power consumption, it has been chosen the ZigBee technology as a simple and a cost-effective solution for remote sensing interrogation and networking. Also an easy-to-use graphical data management interface has been developed using LabWindows platform, allowing continuous sensor monitoring and enhancing automation in industrial processes.

\section{Acknowledgments}

This work has been supported by Spanish CICyT project TEC2009-14718-C03-03 (DEDOS) and by FACTOTEM-2/ 2009/S2009/ESP-1781. The authors want to thank Mercedes Chaparro for her assistance during the wireless sensor network set-up and graphical interface design process.

\section{References}

[1] K. R. Sohn, "Liquid sensors using refractive intensity at the end-face of a glass fiber connected to fiber-Bragg grating," Sensors and Actuators A, vol. 158, no. 2, pp. 193-197, 2010.

[2] K. S. C. Kuang, S. T. Quek, and M. Maalej, "Remote flood monitoring system based on plastic optical fibres and wireless motes," Sensors and Actuators A, vol. 147, no. 2, pp. 449-455, 2008.

[3] M. Lomer, J. Arrue, C. Jauregui, P. Aiestaran, J. Zubia, and J. M. López-Higuera, "Lateral polishing of bends in plastic optical fibres applied to a multipoint liquid-level measurement sensor," Sensors and Actuators A, vol. 137, no. 1, pp. 68-73, 2007.
[4] M. Bottacini, N. Burani, M. Foroni, F. Poli, and S. Selleri, "Allplastic optical-fiber level sensor," Microwave and Optical Technology Letters, vol. 46, no. 6, pp. 520-522, 2005.

[5] F. Pérez-Ocón, M. Rubiño, J. M. Abril, P. Casanova, and J. A. Martínez, "Fiber-optic liquid-level continuous gauge," Sensors and Actuators A, vol. 125, no. 2, pp. 124-132, 2006.

[6] D. S. Montero, C. Vázquez, I. Möllers, J. Arrúe, and D. Jäger, "A self-referencing intensity based polymer optical fiber sensor for liquid detection," Sensors, vol. 9, no. 8, pp. 6446-6455, 2009.

[7] P. Raatikainen, I. V. Kassamakov, R. D. Kakanakov, and M. Luukkala, "Fiber-optic liquid-level sensor," Sensors and Actuators $A$, vol. 58, no. 2, pp. 93-97, 1997.

[8] J. N. Wang and J. L. Tang, "Feasibility of fiber bragg grating and long-period fiber grating sensors under different environmental conditions," Sensors, vol. 10, no. 11, pp. 10105-10127, 2010.

[9] C. Vázquez, A. B. Gonzalo, S. Vargas, and J. Montalvo, "Multisensor system using plastic optical fibers for intrinsically safe level measurements," Sensors and Actuators A, vol. 116, no. 1, pp. 22-32, 2004.

[10] E. J. Chern and B. B. Djordjevic, "Nonintrusive ultrasonic lowliquid-level sensor," Materials Evaluation, vol. 48, no. 4, pp. $481-485,1990$.

[11] K. Shannon, X. Li, Z. Wang, and J. D. N. Cheeke, "Mode conversion and the path of acoustic energy in a partially waterfilled aluminum tube," Ultrasonics, vol. 37, no. 4, pp. 303-307, 1999.

[12] R. Gaudino, D. Cárdenas, M. Bellec et al., "Perspective in nextgeneration home networks: toward optical solutions?" IEEE Communications Magazine, vol. 48, no. 2, pp. 39-47, 2010.

[13] I. F. Akyildiz, X. D. Wang, and W. L. Wang, "Wireless mesh networks: a survey," Computer Networks, vol. 47, no. 4, pp. 445-487, 2005.

[14] D. S. Montero, M. Chaparro, and C. Vázquez, "Wireless mesh network applied to polymer optical fibre-based sensors," in Proceedings of the International Conference on Plastic Optical Fibers (ICPOF '11), Bilbao, Spain, 2011, Paper no. 41. 

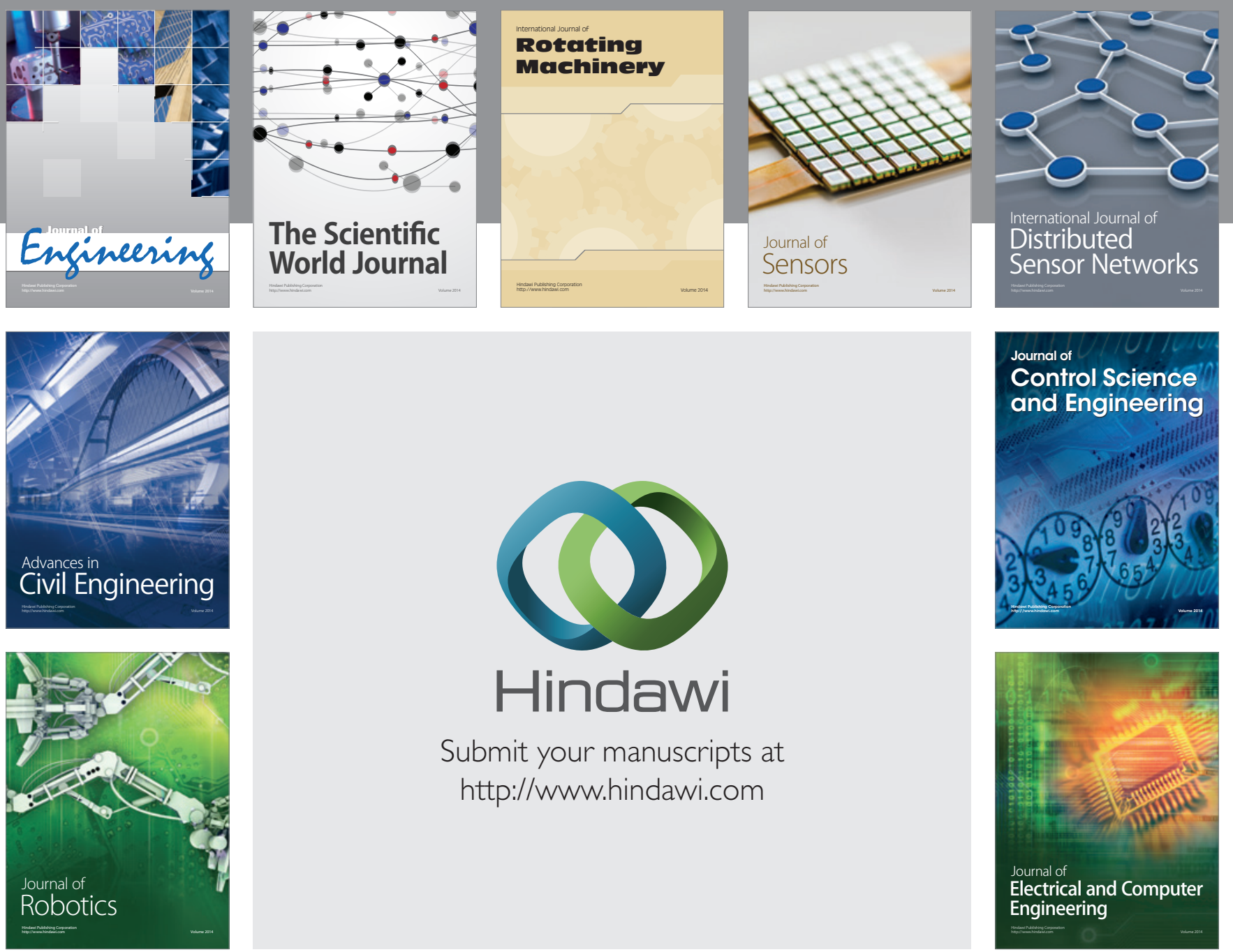

Submit your manuscripts at

http://www.hindawi.com
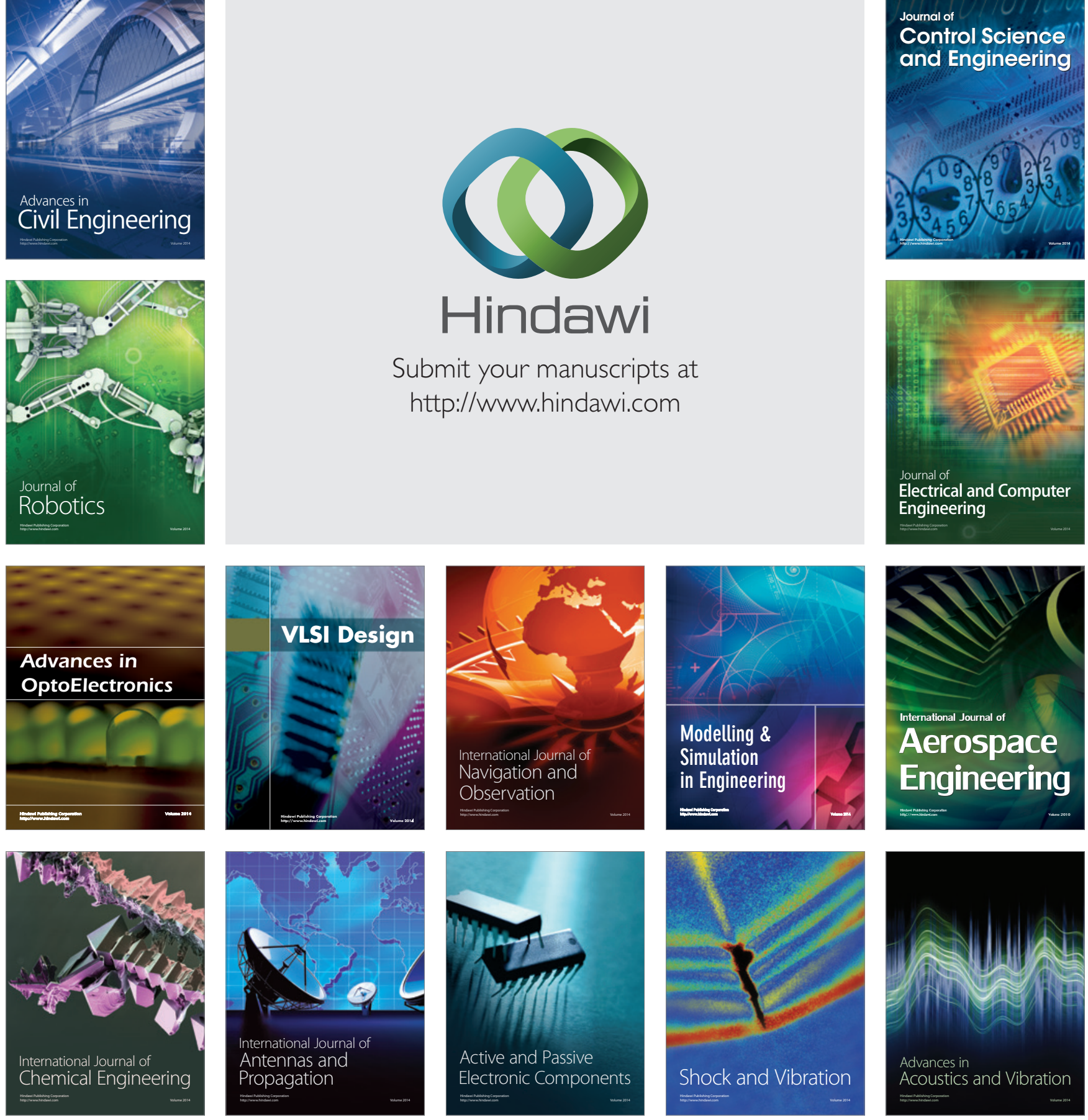